\title{
UNIVERSAL VARIETIES OF SEMIGROUPS
}

\author{
V. KOUBEK and J. SICHLER
}

(Received 7 September 1982)

Communicated by T. E. Hall

\begin{abstract}
A category $\mathbf{V}$ is called universal (or binding) if every category of algebras is isomorphic to a full subcategory of $\mathbf{V}$. The main result states that a semigroup variety $\mathbf{V}$ is universal if and only if it contains all commutative semigroups and fails the identity $x^{n} y^{n}=(x y)^{n}$ for every $n>1$. Furthermore, the universality of a semigroup $v$ riety $\mathbf{V}$ is equivalent to the existence in $\mathbf{V}$ of a nontrivial semigroup whose endomorphism monoid is trivial, and also to the representability of every monoid as the monoid of all endomorphisms of some semigroup in V. Every universal semigroup variety contains a minimal one with this property while there is no smallest universal semigroup variety.
\end{abstract}

1980 Mathematics subject classification (Amer. Math. Soc.): primary 18 B 15, 20 M 07; secondary 20 M 15, 08 A 35 .

\section{Introduction}

Every monoid (that is, a semigroup with an identity element) is isomorphic to the monoid of all endomorphisms of some semigroup and there exist arbitrarily large semigroups with a given endomorphism monoid. This is just one consequence of the universality of the category of all semigroup homomorphisms established in a pioneering article [3] of Z. Hedrlin and J. Lambek; for a somewhat stronger result, see V. Trnková [11], or [10]. From this result and from [12] it also follows that there are rigid semigroups of every infinite cardinality.

These claims obviously fail in small varieties (equational classes) of semigroups: for instance, every element of a semilattice constitutes a one-element subalgebra and hence each constant self-map of a semilattice is one of its endomorphisms. The present note aims to characterize varieties of semigroups that are universal

( 1984 Australian Mathematical Society 0263-6115/84\$A2.00+0.00 
when considered as categories. It is somewhat surprising that this characterization also singles out semigroup varieties representing all monoids as endomorphism monoids of their members.

Recall that a category $\mathbf{A}$ is universal (or binding) if every category of algebras is isomorphic to a full subcategory of $\mathbf{A}$. An object $R$ of $\mathbf{A}$ is rigid if it has only the identity as its endomorphism. Every universal category contains a proper class of nonisomorphic objects with a given endomorphism monoid [5] and hence also a proper class of nonisomorphic rigid objects. For other consequences of universality and a comprehensive presentation of various universality results, the reader is referred to A. Pultr and V. Trnková [7].

To formulate the main result, call a semigroup identity $p(x, \ldots, t)=q(x, \ldots, t)$ balanced if the total degree of each variable $x$ in $p$ equals its total degree in $q$. A semigroup variety $\mathbf{V}$ is balanced if it is definable by balanced identities alone. Thus, for instance, the variety $\mathbf{C}$ of commutative semigroups is balanced and, in fact, a semigroup variety is balanced if and only if it contains $\mathbf{C}$. Commutative semigroups do not form a binding category since the $n$th power law $x^{n} y^{n}=(x y)^{n}$ valid in $\mathbf{C}$ for all positive $n$ implies that the mapping assigning $x^{n}$ to every element $x$ of any such semigroup is one of its endomorphisms.

THEOREM 1.1. A semigroup variety $\mathrm{V}$ is binding if and only if it is balanced and fails the nth power law for every $n>1$.

From a point of view of universal algebra Theorem 1.1 appears to be one of the few structural characterizations of binding subvarieties of a given variety; an early example of a result of this type concerning unary varieties can be found in [6]. The only complete characterization of binding unary varieties [8] uses categorical terms.

Since the non-universality of a semigroup variety manifests itself already on its one-object subcategories, the following characterization is also obtained.

THEOREM 1.2. For any semigroup variety $\mathbf{V}$ the following are equivalent:

(1) $\mathrm{V}$ contains a nontrivial rigid semigroup,

(2) $\mathbf{V}$ has arbitrarily large rigid objects,

(3) for any monoid $M$ the variety $\mathbf{V}$ contains arbitrarily large semigroups with endomorphisms monoids isomorphic to $M$.

(4) $\mathrm{V}$ is universal.

A variety is group-universal if every group is isomorphic to the full automorphism group of an algebra from $\mathbf{V}$. Every universal variety is also group-universal. 
It may be of some interest to point out that [2] describes all group-universal varieties of semigroups (for example, semilattices form such a variety), and that group-universal unary varieties are characterized by [9].

\section{Equationally definable semigroup homomorphisms}

This section establishes a necessary condition for universality and characterizes varieties satisfying an $n$th power law.

LEMMA 2.1. Any semigroup variety which is not balanced contains only trivial rigid semigroups.

Proof. If the total degree of $p$ differs from that of $q$, then an identity $x^{m+n}=x^{m}$ with $m, n>0$ is obtained if all variables of $p=q$ are identified. If the total degrees of $p$ and $q$ both equal $t$ and $x$ occurs $r$ times in $p$, substitute $x^{2}$ for all variables of $p=q$ distinct from $x$ to arrive at an identity $x^{2 t-r}=x^{2 t-s}$ in which $s$ is the total degree of $x$ in $q$. We see that an identity of the form $x^{m+n}=x^{m}$ with $m, n>0$ follows from any non-balanced semigroup identity. Now $x^{m+m n}=x^{m}$, and the identity $x^{2 m n}=x^{m n}$ is obtained; the constant mapping whose value is $x^{m n}$ is an endomorphism of any semigroup satisfying $p=q$. Such a semigroup is rigid only if it is trivial.

LEMMA 2.2. No nontrivial semigroups satisfying an nth power law for some $n>1$ are rigid. Moreover, there are monoids not representable as endomorphism monoids of such semigroups.

Proof. If $x^{n} y^{n}=(x y)^{n}$ in a semigroup $S$, then the mapping $f(x)=x^{n}$ is an endomorphism of $S$ commuting with all other endomorphisms of $S$. Whenever the center of the monoid to be represented by endomorphisms of $S$ is trivial, the nonbalanced identity $x^{n}=x$ must be satisfied in $S$ and hence $S$ has a constant endomorphism with the value $x^{n-1}$ for each element $x$ of $S$. Therefore no monoid without left zeros whose center is trivial can be represented and, in particular, each rigid semigroup $S$ satisfying the $n$th power law for some $n>1$ is trivial.

Since every binding category contains a proper class of nonisomorphic rigid objects the claim below follows immediately.

COROLlary 2.3. If a semigroup variety $\mathbf{V}$ does not contain $\mathbf{C}$ or if it satisfies the nth power law for some $n>1$, then there exist monoids not occurring as endomorphism monoids of semigroups from $\mathbf{V}$; thus such a variety is not universal. 
From the properties of categorical universality mentioned earlier it is now possible to conclude the validity of the implications $(4) \rightarrow(3) \rightarrow(2) \rightarrow(1)$. It is therefore enough to show that every balanced semigroup variety $\mathbf{V}$ failing the $n$th power law for every $\boldsymbol{n}>\boldsymbol{1}$ is universal. First we characterize these varieties.

Let $V(\{a, b, c, d, e\})$ denote the semigroup freely generated by $\{a, b, c, d, e\}=$ $A$ in a balanced variety $\mathbf{V}$. Assume that for the least congruence $\theta(a b c, d e)$ containing the pair $\{a b c, d e\}$

(5) $\left\{a^{n} b^{n} c^{n}, d^{n} e^{n}\right\} \in \theta(a b c, d e)$ for some $n>1$;

note that (5) holds if $\mathbf{V}$ satisfies the $n$th power law. If $f$ is a homomorphism of $V(A)$ into $V(\{x, y, z\})$ such that $f(a)=f(d)=x, f(b)=y, f(c)=z$, and $f(e)=$ $y z$, then the kernel of $f$ identifies $a b c$ with $d e$. By (5) we obtain $x^{n} y^{n} z^{n}=x^{n}(y z)^{n}$, so that

(6) $x^{n}(y z)^{n}=x^{n} y^{n} z^{n}=(x y)^{n} z^{n}$ are identities of $\mathbf{V}$, where the second identity follows similarly. Furthermore,

(7) for $N=n^{2}$, both $x^{n} y^{N} z^{N}=x^{n}(y z)^{N}$ and $x^{N} y^{N} z^{n}=(x y)^{N} z^{n}$ are identities of $\mathbf{V}$.

To prove the first identity set $v=y^{n}, t=z^{n}$; then

$$
\begin{aligned}
x^{n} y^{N} z^{N} & =x^{n} v^{n} t^{n}=x^{n}(v t)^{n}=x^{n}\left(y^{n} z^{n}\right)(v t)^{n-1} \\
& =x^{n}(y z)^{n}(v t)^{n-1}=x^{n}(y z)^{n} y^{n} z^{n}(v t)^{n-2}=x^{n}(y z)^{2 n} y^{n} z^{n}(v t)^{n-3} \\
& =\cdots=x^{n}(y z)^{(n-1) n} y^{n} z^{n}=x^{n}(y z)^{N}
\end{aligned}
$$

follows by repeated applications of (6). In addition to (5), suppose

(8) $\left\{a^{r} b^{r}, d^{r} e^{r}\right\} \in \theta(a b, d e)$ for some $r>1$.

The congruence $\theta(a b, d e)$ is the transitive closure of the set of pairs of the form $\{B a b C, B d e C\}$ with (possibly empty) words $B, C$ in $a, b, c, d, e$. Since $V(A)$ is a free algebra in a balanced variety, the total degree of $U$ equals that of $W$ whenever $\{U, T\},\{T, W\}$ are two such pairs. Thus if (8) holds, then all elements occuring in pairs $\{B a b C, B d e C\}$ of any string connecting $a^{r} b^{r}$ to $d^{r} e^{r}$ have total degree $2 r>2$; in particular, at least one of $B, C$ is a nonempty word. Consider a homomorphism $g$ of $V(A)$ into $V(\{x, y\})$ defined by $g(a)=x^{N}, g(b)=y^{N}$, $g(c)=g(d)=(x y)^{n}, g(e)=(x y)^{n(n-1)}$. Using (7) it is easily verified that $g(t) g(a b)=g(t) g(d e)$ and $g(a b) g(t)=g(d e) g(t)$ for all $t \in A$; since at least one of the words $B, C$ is nonempty, it follows that $g(B a b C)=g(B d e C)$ for each pair $\{B a b C, B d e C\}$ contained in any string connecting $a^{r} b^{r}$ to $d^{r} e^{r}$. Therefore $x^{N r} y^{N r}=g\left(a^{r} b^{r}\right)=g\left(d^{r} e^{r}\right)=(x y)^{N r}$ with $N r>1$. This proves the characterization below, for the converse implication is trivial.

Proposition 2.4. A balanced semigroup variety $\mathrm{V}$ satisfies the $k$ th power law for some $k>1$ if and only if (5) and (8) hold in the five-generated $\mathbf{V}$-free semigroup. 
The failure of (5) or of (8) will be used to construct two full embeddings of a category of graphs into $\mathbf{V}$ in the last section. In addition, extensions of semigroups by elements of infinite prime height will be employed in a manner analogous to that used by $\mathrm{L}$. Fuchs to build large indecomposable abelian groups in [1].

\section{Elements of infinite $p$-height}

Throughout this section, let $\mathbf{V}$ be an arbitrary balanced semigroup variety. For an arbitrary nonempty set $Y$, let $V(Y)$ denote the semigroup freely generated by $Y$ in $\mathrm{V}$, and let $R$ denote the additive semigroup of all non-negative rational numbers. Note that $R$ lies in the variety $\mathbf{V}$ by virtue of its commutativity. Let $N$ denote the set of all positive integers.

An element $s$ of a semigroup $S$ is said to have an infinite p-height in $S$ if there are elements $s=s_{0}, s_{1}, \ldots, s_{i}, \ldots$ such that $\left(s_{i+1}\right)^{p}=s_{i}$ for all non-negative integers $i$.

For an arbitrary subset $W$ of $V(Y)$ let $Z$ be the union of $Y$ with $W \times N$. Let $P$ be an arbitrary mapping of $W$ into the set of all prime numbers, and let $S$ denote the quotient of $V(Z)$ modulo the least congruence $\theta$ satisfying

(9) $(w, i+1)^{P(w)} \theta(w, i)$ for all $(w, i) \in W \times N$,

(10) $(w, 1)^{P(w)} \theta w$ for all $w \in W$.

There exists a homomorphism $h$ of $V(Z)$ into $R^{Y}$ such that, for $x, y \in Y$, $[h(y)](x)=0$ if $y \neq x,[h(y)](y)=1$, and $h(w, i)=P(w)^{-i} h(w)$ for $(w, i) \in W$ $\times N$. It is easy to verify that the kernel of $h$ satisfies (9) and (10); from the definition of $S$ it now follows that there exists a homomorphism $t: S \rightarrow R^{Y}$ such that $h=t \circ f$ where $f$ is the canonical homomorphism with $\operatorname{Ker}(f)=\theta$. Clearly, all but finitely many components $[t(s)](y)$ of $t(s)$ vanish, and for every prime number $p$ the sequence $t(s)$ is of infinite $p$-height whenever $s$ is.

Let $W_{p}$ be the set of all $w \in W$ with $P(w)=p$; we aim to show that all elements of $S$ with infinite $p$-height lie in a subsemigroup $S_{p}$ of $S$ generated by $f\left(W_{p} \times N\right)$.

To this end, let $s \in S$ have infinite $p$-height. If $a$ is an upper bound of $[t(s)](y)$ for $y \in Y$, choose an integer $k$ large enough to satisfy $p^{k}>a$. Since $t(s)=p^{k} t\left(s_{k}\right)$ for the $p^{k}$ th root $s_{k}$ of $s$, all values of $t\left(s_{k}\right)$ are positive rationals smaller than one, and thus we may assume that

(11) $[t(s)](y)<1$ for all $y \in Y$.

Note that every $s$ satisfying (11) belongs to the subsemigroup [ $f(W \times N)]$ of $S$ generated by $f(W \times N)$. 
Assume that $s=f(u(w, j) v)$ for some $u, v \in V(Z)$ and let $P(w)=q \neq p$. Choose $y \in Y$ occurring in $w$. Then $[t(s)](y)=a q^{-j}+b$ with a nonzero integer $a$ and a $q$-integer $b$. If $s_{k}$ is a $p^{k}$ th root of $s$, then $t(s)=p^{k} t\left(s_{k}\right)$, and $\left[t\left(s_{k}\right)\right](y)=$ $a_{k} q^{-i}+b_{k}$ for an integer $a_{k}$ and a $q$-integer $b_{k}$. Hence $a q^{-j}+b=p^{k} a_{k} q^{-i}+p^{k} b_{k}$; since this rational and all the additive terms of the latter equality are between 0 and $1, a q^{-j}=p^{k} a_{k} q^{-i}$ follows. Thus $a$ is divisible by arbitrarily high powers of $p$; this contradiction shows that every element of $S$ whose $p$-height is infinite must belong to $S_{p}$.

LEMMA 3.1. Any element of $S$ of infinite p-height belongs to $S_{p}=f\left[W_{p} \times N\right]$.

For every $v \in V(Z)$ set $v(v)=\{y \in Y ;[h(v)](y)>0\}$; note that $c(y)=\{y\}$ for all $y \in Y, c(w)=c(w, i)$ for all $(w, i) \in W \times N$ and $c\left(v_{1} v_{2}\right)=c\left(v_{1}\right) \cup c\left(v_{2}\right)$ if $v_{i} \in V(Z)$. It is easily seen that $c$ maps $V(Z)$ onto the semilattice $S(Y)$ freely generated by $Y$ and that $c$ has $h$ (and hence $f$ ) as its left factor.

Let $A \subseteq Y$ and assume that both $r, s \in[A]$ are products of at least two distinct elements of $A$. Furthermore, let

(12) $c(r) \cap c(s)=\varnothing$,

(13) $c(w) \subseteq A$ for no $w \in W$,

(14) $c$ be one-to-one on $W$,

(15) $c(r), c(s) \subseteq c\left(W_{p}\right)$ for no prime $p$, where $c\left(W_{p}\right)=\cup\left(c(w): w \in W_{p}\right)$. From (13) we immediately obtain

(16) $c(v) \subseteq A$ only if $v \in[A]$.

Set $S(r, s)=V(Z) /(\theta(r, s) \vee \theta)=S / \theta(f(r), f(s))$ and let $g$ denote the corresponding homomorphism of $V(Z)$ onto $S(r, s)$.

LEMMA 3.2. If $r, s$ are products of at least two distinct elements of $A \subseteq Y$ and if (12)-(15) hold, then

(a) $g$ is one-to-one on $Z=Y \cup(W \times N)$,

(b) $g[A]$ is isomorphic to $V(A) / \theta(r, s)$,

(c) $g(v) \in g\left[W_{p} \times N\right]$ only if $c(v) \subseteq c\left(W_{p}\right)$,

(d) every element of $S(r, s)$ with infinite $p$-height is contained in $g\left[W_{p} \times N\right]$,

(e) $g(w, i)$ is the only $P(w)^{i}$ th root of $w \in W$ that has infinite $P(w)$-height.

Proof. Every nontrivial generating pair of the congruence $\theta$ contains an element $v$ such that $c(w) \subseteq c(v)$ for some $w \in W$; since $c$ is constant on the classes of $\theta$, we see that

(*) if $u \theta v$ are distinct, then there is a $w \in W$ such that $c(w) \subseteq c(u)=c(v)$.

Similarly,

(**) if $u$ is an element of a nontrivial class of $\theta(r, s)$ then $c(r) \subseteq c(u)$ or $c(s) \subseteq c(u)$. 
In view of (*) and (13) only trivial classes of $\theta$ intersect $[A]$. If $\{B r C, B s C\}$ is a generating pair of $\theta(r, s)$, then $B r C \in[A]$ yields $c(B) \cup c(C) \subseteq A$, so that $B s C \in[A]$ by $(16)$; thus each class of $\operatorname{Ker}(g)$ intersecting $[A]$ is, in fact, a class of the least congruence $\theta(r, s)$ on $V(A)$ identifying $r$ with $s$. This proves (b).

Similarly, (**) and (15) show that all classes of $\theta(r, s)$ intersecting any $\left[W_{p} \times N\right]$ are trivial. For a nontrivial pair $\{u, v\} \in \theta$ with $u \in\left[W_{p} \times N\right]$ we obtain $c(v) \subseteq c\left(W_{p}\right)$ by (*); if, in addition, $\{v, t\} \in \theta(r, s)$, then a contradiction with (15) would follow from (*) if $v \neq t$. This proves (c). If $y \in Y,\{y, v\} \in \theta$ and $\{v, t\} \in \theta(r, s)$ is a nontrivial pair, then $c(r) \subseteq c(v)=c(y)=\{y\}$ or $c(s) \subseteq\{y\}$ follow from (*) and (**), contradicting the choice of $r, s$. Altogether, the kernels of $f$ and that of $g$ coincide on $Y \cup \cup\left(\left[W_{p} \times N\right]: p\right)$; from (14) and the definition of $h$ we conclude that $h$, and therefore also $f$ are one-to-one on $Y \cup(W \times N)$. This concludes the proof of (a); note also that $g\left[W_{p} \times N\right]$ is isomorphic to $S_{p}$.

Let $E: S \rightarrow S(r, s)$ be the homomorphism whose kernel is $\theta(f(r), f(s))$. If $a_{0} \in S(r, s)$ has infinite $p$-height, then there are $b_{i} \in S$ with $E\left(b_{i+1}\right)^{p}=E\left(b_{i}\right)$, $E\left(b_{0}\right)=a_{0}$. To prove $(d)$, define an auxiliary function $M: S \rightarrow R$ by

$$
M(b)=\max \{[t(b)](y): y \in c(r)\}+\max \{[t(b)](z): z \in c(s)\} .
$$

If $\{d f(r) e, d f(s) e\}$ is a generating pair of $\theta(f(r), f(s))$, then $M(d f(r) e)=M(d e)$ $+1=M(d f(s) e)$ by (12) and the choice of $r, s$; hence $M$ is constant on each nontrivial class of $\theta(f(r), f(s))$ and its value is at least one on such a class. Note also that $M\left(b^{k}\right)=k M(b)$ for every positive integer $k$. Hence $M(b)=p^{i} M\left(b_{i}\right)>p^{i}$ whenever $b_{i}$ lies in a nontrivial class of $\theta(f(r), f(s))$, and this is possible only if there is an integer $j$ such that all $b_{i}$ with $i>j$ lie in trivial classes of $\theta(f(r), f(s))$. Therefore $b_{i}$ is the $p$ th power of $b_{i+1}$ for all $i>j$ and Lemma 3.1 gives $b_{i} \in f\left[W_{p} \times N\right]$. Hence $E\left(b_{i}\right) \in g\left[W_{p} \times N\right]$ and (d) follows since $a_{0} \in\left[E\left(b_{i}\right)\right]$ for all $i$.

If $g(v)$ is a $p^{i}$ th root with infinite $p$-height of $g(w)$ for $w \in W$, then $g(v) \in$ $g\left[W_{p} \times N\right]$ by (d). Since this subsemigroup is isomorphic to $S_{p}, c(v)=c(w)$ and (14) implies that $v=(w, j)^{k}$ since each $[\{w\} \times N]$ is commutative. Now $h(w)=$ $p^{i} h(v)=p^{i-j} k h(w)$, so that $k=p^{j-i}$ and (e) is proved.

Thus we may assume that, under the hypothesis of Lemma 3.2, $S(r, s)$ is generated by $Y \cup(W \times N)$ and that $A \subseteq Y$ generates $V(A) / \theta(r, s)$ in $S(r, s)$.

\section{The construction}

An undirected graph is pair $(X, R)$ in which $R$ is a set of two-element subsets of the set $X$. The category $\mathbf{G}$ of all connected undirected graphs and their compatible maps (that is, maps $f: X \rightarrow X^{\prime}$ with $\{f(x), f(y)\} \in R^{\prime}$ for each $\{x, y\} \in R$ ) is 
binding [4]. To prove that a semigroup variety $\mathbf{V}$ is binding it is enough to construct a full and faithful functor $F: \mathbf{G} \rightarrow \mathbf{V}$; recall that $F$ is full if every morphism $v: F(G) \rightarrow F\left(G^{\prime}\right)$ is of the form $v=F(g)$ for some morphism $g$ : $G \rightarrow G^{\prime}$ of $\mathbf{G}$, and faithful if it is one-to-one on each $\operatorname{Hom}\left(G, G^{\prime}\right)$.

From now on, let $\mathbf{V}$ be a balanced semigroup variety failing the $k$ th power law for every $k>1$.

Let $B=\{a, b, c, d, e, u, v\}, A=B \backslash\{u, v\}$, and let $(X, R) \in \mathbf{G}$. Set $Y(X)=Y$ to be the disjoint union of $X$ with $B$. For $i=1, \ldots, 10$ let $W_{i}=\{k l\}$, where $k l$ is the product of $k \in A$ with $l \in\{u, v\}$ in $V(Y)$ and the singleton sets $W_{i}$ are pairwise disjoint. Furthermore, let $W_{11}$ consist of all products $u x$ with $x \in X$, and set $W_{12}=\{v x y:\{x, y\} \in R\}$. Let $W=\cup\left(W_{i}: i=1, \ldots, 12\right)$, select distinct prime numbers $p_{i}$ and define a mapping $P$ on $W$ by $P(w)=p_{i}$ for $w \in W_{i}$. Next, let $r_{0}=a b, r_{1}=a b c, s=d e$ and note that these elements satisfy the hypotheses of Lemma 3.2. Denote $\theta_{j}=\theta\left(r_{j}, s\right)$ for $j=0,1$ and define $F_{j}(X, R)$ as the quotient of $V(Y \cup(W \times N))$ modulo the least congruence $\theta_{j}(X, R)$ containing both $\theta_{j}$ and $\theta$ defined by (9) and (10).

For a compatible mapping $f:(X, R) \rightarrow\left(X^{\prime}, R^{\prime}\right)$ define $f^{+}$as $f$ on $X, f^{+}(t)=t$ for $t \in B$; if $V\left(f^{+}\right)$is the free extension of $f^{+}$to the semigroup $V(Y)$, define $f^{*}$ : $V(Z) \rightarrow V\left(Z^{\prime}\right)$ by $f^{*}(w, i)=\left(V\left(f^{+}\right)(w), i\right), f^{*}(v)=V\left(f^{+}\right)(v)$ on $V(Y)$. Only a routine computation is needed to verify that $f^{*}$ maps each class of $\theta_{j}(X, R)$ into a class of $\theta_{j}\left(X^{\prime}, R^{\prime}\right)$; hence there is a semigroup homomorphism $F_{j}(f): F_{j}(X, R) \rightarrow$ $F_{j}\left(X^{\prime}, R^{\prime}\right)$. It is also easily seen that $F_{j}$ is a functor; since (12)-(15) are satisfied, Lemma 3.2(a) applies and hence $F_{j}$ is a faithful functor for $j=0,1$.

Next we shall consider a semigroup homomorphism $H: F_{j}(X, R) \rightarrow F_{j}\left(X^{\prime}, R^{\prime}\right)$. Let $k \in A$; there are distinct primes $p, q$ such that $(k u, 1),(k v, 1)$ are of infinite $p$-height, $q$-height respectively. Hence $H(k u, 1)$ has infinite $p$-height; from the definition of $F_{j}\left(X^{\prime}, R^{\prime}\right)$ and from Lemma 3.2(d) we conclude that $H(k u, 1)$ is a power of some $(k u, m)$. Similarly, $H(k v, 1)$ is a power of some $(k v, n)$. From $k u=(k u, 1)^{p}$ it follows that $H(k)$ is a factor of some power of $(k u, m)$ and, analogously, a factor of a power of $(k v, n)$. Hence $c(t) \subseteq c\left(W_{p}\right) \cap c\left(W_{q}\right)=\{k\}$ for any $t$ with $g(t)=H(k)$ by Lemma 3.2(c); in other words, $H(k)$ is a power of $k$. A similar argument applies to $u$ and $v$; thus $H(k)$ is a power of $k$ for all $k \in B$. If, say, $H(a)=a^{n}$ and $H(u)=u^{r}$ then $a^{n} u^{r}=H(a u)$ has infinite $p$-height only if it equals to a power of some $(a u, i)$ : see (d) of Lemma 3.2 and note that $W_{p}=\{a u\}$; this is possible only if $n=r$. Since this argument applies to all pairs from $A \times\{u, v\}$ we conclude the existence of a positive $n$ such that $H(k)=k^{n}$ for all $k \in B$. In particular, $a^{n} b^{n}=d^{n} e^{n}$ must hold if $j=0$ and $a^{n} b^{n} c^{n}=d^{n} e^{n}$ for $j=1$. In view of Proposition 2.4 and (b) of Lemma 3.2 there is a $j$ such that $n=1$ is the only possibility, that is, the homomorphism $H: F_{j}(X, R) \rightarrow F_{j}\left(X^{\prime}, R^{\prime}\right)$ fixes all elements of $[B]$. 
Every $x \in X$ is contained in some $\{x, y\} \in R$ and hence $u x \in W_{11}, v x y \in W_{12}$; there are distinct primes $p, q$ such that $u x$ has infinite $p$-height and $v x y$ has infinite $q$-height. From Lemma 3.2(d) it follows that $u H(x)=H(u x) \in g\left[W_{p} \times\right.$ $N]$ and $v H(x) H(y) \in g\left[W_{q} \times N\right]$. Now $H(x)$ is a factor of both of these elements, so that $c(t) \subseteq c\left(W_{11}\right) \cap c\left(W_{12}\right)=X^{\prime}$ for every $t$ satisfying $H(x)=g(t)$ follows from Lemma 3.2(c). Therefore $H(x)=x_{1} x_{2} \cdots x_{k}$; on the other hand, $u x_{1} x_{2} \cdots x_{k} \in g\left[W_{11} \times N\right]$ only if $k=1$. We conclude that $H(x) \in X^{\prime}$, so that the restriction $f$ of $H$ to the subset $X$ of $Z=X \cup B \cup(W \times N)$ maps $X$ into $X^{\prime}$. Finally, an element $v f(x) f(y)=H(v x y)$ is of infinite $q$-height only if $\{f(x), f(y)\} \in R^{\prime}$, thus $f$ is a compatible mapping of $(X, R)$ into $\left(X^{\prime}, R^{\prime}\right)$. In fact, $H$ coincides with $f^{+}$on $Y=X \cup B$. Since $H$ preserves $W_{k} \subseteq[Y]$ for every $k$, it easily follows from Lemma 3.2(e) that $H=F_{j}(f)$; this shows that the functor $F_{j}$ is full and thus finishes proofs of both theorems.

\section{Concluding remarks}

There is no smallest universal semigroup variety: the intersection of varieties given by $x y z t=y x z t, x y z t=x y t z$ respectively satisfies the third lower law, while any $(x y)^{n}$ can only be equal to a polynomial of the form $A x y, x y A$ respectively, with $A$ of total degree $2(n-1)$, so that every nontrivial $n$th power law fails in these balanced varieties. Each semigroup variety $\mathbf{V}_{n}$ given by the single identity $x^{n} y^{n}=(x y)^{n}$ for $n>1$ is a nonextremal dually compact element in the dually algebraic lattice of all semigroup varieties. The intersection of a chain of varieties not contained in any $\mathbf{V}_{n}$ cannot be contained in any $\mathbf{V}_{n}$ either. In view of the presented characterization, Zorn's Lemma shows that every universal semigroup variety contains a minimal universal one. This partially solves the semigroup case of Problem 7 posed by A. Pultr and V. Trnková [7]; a complete solution should characterize minimal binding varieties structurally.

\section{References}

[1] L. Fuchs, Infinite abelian groups, Volume II (Academic Press, 1973).

[2] P. Goralčík and V. Koubek, 'Minimal group universal varieties of semigroups', to appear.

[3] Z. Hedrlin and J. Lambek, 'How comprehensive is the category of semigroups', J. Algebra 11 (1969), 195-212.

[4] Z. Hedrlin and A. Pultr, 'Relations (graphs) with given infinite semigroups', Monatsh. Math. 68 (1964), 421-425.

[5] Z. Hedrlin and J. Sichler, 'Any boundable binding category contains a proper class of mutually disjoint copies of itself', Algebra Universalis 1 (1971), 97-103. 
[6] A. Pultr and J. Sichler, 'Primitive classes of algebras with two unary idempotent operations, containing all algebraic categories as full subcategories', Comment. Math. Univ. Carolinae 10 (1969), 425-445.

[7] A. Pultr and V. Trnková, Combinatorial, algebraic and topological representations of groups, semigroups and categories (North-Holland, 1980).

[8] J. Sichler, 'Testing categories and strong universality', Canad. J. Math. 25 (1973), 370-385.

[9] J. Sichler, 'Group universal unary varieties', Algebra Universalis 11 (1980), 12-21.

[10] J. Sichler, ' $A(1,1)$ can be strongly embedded into the category of semigroups', Comment. Math. Univ. Carolinae 9 (1968), 257-262.

[11] V. Trnková, 'Strong embedding of category of all groupoids into category of semigroups', Comment. Math. Univ. Carolinae 9 (1968), 251-256.

[12] P. Vopěnka, A. Pultr and Z. Hedrlín, 'A rigid relation exists on any set', Comment. Math. Univ. Carolinae 6 (1965), 149-155.

\section{MFF KU}

Malostranské nám. 25

Praha 1

Czechoslovakia
Department of Mathematics University of Manitoba Winnipeg, Manitoba Canada R3T 2N2 